\title{
AN EXAMPLE IN SHAPE THEORY
}

\author{
SMILKA ZDRAVKOVSKA ${ }^{1}$
}

\begin{abstract}
We give an example of a compactum which cannot be "improved" within its shape class so that its shape theory agrees with its homotopy theory.
\end{abstract}

1. Introduction. S. Ferry [2] has proved the following theorem: For every $U V^{(1)}$ compactum $X$ there is an "improved" compactum $X^{\prime}$ shape equivalent to $X$ such that for every finite-dimensional compactum $Z$ there is a one-to-one correspondence between the set of homotopy classes and the set of strong shape morphisms from $Z$ to $X^{\prime}$. Two questions arise concerning the hypotheses in that theorem:

(i) Is $U V^{(1)}$ a necessary requirement; i.e. is there a compactum that cannot be improved?

(ii) Is the finite dimensionality of $Z$ essential?

In this paper we give an example to answer in the affirmative the first question.

I have to thank Ross Geoghegan for introducing me to Ferry's work and for his interest and help. In particular, he communicated a version of the folk lemma given in the Appendix. The referee suggested the version he wanted to see published and expressed regret Chapman had not done so in 1970. I am grateful to the referee for changing the original example (so that one would not be misled by the size of $\pi_{1}$ ), the Hawaiian earring.

2. The example. Let $X_{n}$ denote the wedge of a circle with $\boldsymbol{n}$ copies of the $\boldsymbol{k}$-sphere $S^{k}, n \geqslant 0, k \geqslant 2$. Let $X$ be the limit of the sequence $X_{0} \leftarrow X_{1} \leftarrow \cdots$ with bond $p_{m}^{n}: X_{n} \rightarrow X_{m}$ equal to the identity on $S^{1}$ and the first $m$ copies of $S^{k}$, and sending the remaining $n-m$ copies of $S^{k}$ to the basepoint.

Note that $X_{n}$ inherits a metric as a subset of $S^{1} \vee B^{k+1} \subset S^{1} \times B^{k+1}$, where $B^{k+1}$ is the $(k+1)$-dimensional ball.

THEOREM. For any compactum $Y$ shape equivalent to $X$, the natural map

$$
\left[S^{k}, Y\right]_{*} \rightarrow\left\{\text { strong shape morphisms from } S^{k} \text { to } Y\right\}_{*}
$$

is not surjective.

To get an idea of the proof, consider first the case $Y=X$. Let $a_{1}, \ldots, a_{n}$ be the obvious generators of $\pi_{k}\left(X_{n}\right)$ as a module over $\pi_{1}\left(X_{n}\right)=\mathbf{Z}$ (i.e. $a_{i}$ is represented by

Received by the editors June 12, 1980 and, in revised form, November 3, 1980.

1980 Mathematics Subject Classification. Primary 55P55; Secondary 57N25.

Key words and phrases. Shape equivalence, shape morphism, Hilbert cube manifold.

${ }^{1}$ The author gratefully acknowledges the hospitality of the University of Florida Mathematics Department and of Jed Keesling in particular. 
a map that sends $S^{k}$ by the identity to the $i$ th copy of $S^{k}$ in $\left.X_{n}\right)$. We choose a generator $t$ of $\pi_{1}\left(X_{n}\right)$ and denote the action of $t$ on $\pi_{k}\left(X_{n}\right)$ by $g \mapsto t g$. Any infinite word of the form

$$
a=\sum_{i=1}^{\infty}\left(t^{k_{i}}+t^{-k_{i}}\right) a_{i}
$$

with $k_{i} \rightarrow \infty$ as $i \rightarrow \infty$, determines a shape morphism $S^{k} \rightarrow X$ which cannot be represented by a map. Since there is a forgetful map from strong shape morphisms to shape morphisms, this suffices to prove the theorem.

Let $A$ denote the set of all such $a$ 's. It is the size of $A$ that makes the theorem true. For, given any finite subset $A^{\prime} \subset A$, there is a compactum $X^{\prime}$ and a shape equivalence $h: X \rightarrow X^{\prime}$ such that for every $a \in A^{\prime}$, ha is representable by a map: just take $X^{\prime}$ to be the shape mapping cylinder of $\bigsqcup_{a \in A^{\prime}} a: \bigsqcup_{a \in A^{\prime}} S^{k} \rightarrow X$.

Proof of THE THEOREM. Let $Y$ be a compactum shape equivalent to $X$. It follows from the Appendix that there is a sequence of embeddings $j_{n}^{n+1}: X_{n+1} \times Q \rightarrow$ $X_{n} \times Q(Q=$ Hilbert cube $)$ such that

$$
Y=\lim _{\leftarrow}\left(X_{0} \times Q \stackrel{j_{0}^{1}}{\leftarrow} X_{1} \times Q \stackrel{j_{1}^{2}}{\leftarrow} \cdots\right)=\bigcap_{n=0}^{\infty} j_{0}^{n}\left(X_{n} \times Q\right)
$$

and such that $j_{n}^{n+1}$ is homotopic to $p_{n}^{n+1} \times \mathrm{id} Q$. The natural map from $Y$ to $Y_{n}=X_{n} \times Q$ will be denoted by $j_{n}$.

If $\alpha: S^{k} \rightarrow Y$ is a map, then $j_{0} \alpha=j_{0}^{n} j_{n} \alpha: S^{k} \rightarrow Y_{0}=S^{1} \times Q$ is null-homotopic and lifts to the universal cover $\mathbf{R}^{1} \times Q$ of $S^{1} \times Q$. We will construct a shape morphism $\omega: S^{k} \rightarrow Y$ such that if $\omega_{n}: S^{k} \rightarrow Y_{n}$ is any map representing the shape morphism $j_{n} \omega$, then the sequence diam $\widetilde{j_{0}^{n} \omega_{n}}\left(S^{k}\right)$ is unbounded, where $\widetilde{j_{0}^{n} \omega_{n}}$ is any lift of $j_{0}^{n} \omega_{n}$ to the universal cover $\mathbf{R}^{1} \times Q$ of $S^{1} \times Q$. This shows that $\omega$ is not representable by a map.

Let $\tilde{Y}_{n}$ denote the universal cover of $Y_{n}$. Note that $\overparen{X_{n} \times Q}=\tilde{X}_{n} \times Q$ has a metric which agrees locally with the metric on $X_{n} \times Q$, is invariant under covering translations, and agrees with the usual metric on $\mathbf{R}^{1} \times B^{k+1} \times Q$. In particular, if $f: A \rightarrow X_{n}$ is a map, then $\operatorname{diam} \tilde{f}(A)$ is independent of the choice of the lifting $\tilde{f}$ : $A \rightarrow \tilde{X}_{n}$ of $f$, provided $A$ is path connected.

LEMMA. If the map $f: X_{n} \times Q \rightarrow X_{0} \times Q$ is such that

$$
f_{*}: \pi_{1}\left(X_{n} \times Q\right) \rightarrow \pi_{1}\left(X_{0} \times Q\right)
$$

is an isomorphism, then for each $N>0$ there exists an $M>0$ such that if $A \subset \tilde{X}_{n} \times Q$ is a subset with $\operatorname{diam} A>M$ and $\tilde{f}: \tilde{X}_{n} \times Q \rightarrow \tilde{X}_{0} \times Q$ covers $f$, then $\operatorname{diam} \tilde{f}(A)>N$.

Proof. This follows immediately from the fact that $f$ is proper and from our choice of metrics.

We will now construct the desired shape morphism $\omega: S^{k} \rightarrow Y$. It will be determined by an infinite word of the form $\omega=\sum_{i=1}^{\infty}\left(t^{k_{i}}+t^{-k_{i}}\right) a_{i}$. Each such word defines a shape morphism $\omega: S^{k} \rightarrow Y$ such that $j_{n} \omega$ is represented by $\left[\omega_{n}\right]=$ $\sum_{i=1}^{n}\left(t^{k_{i}}+t^{-k_{i}}\right) a_{i}$. Choose $M_{n}$ as in the lemma above so that if $\operatorname{diam} A>M_{n}$, then 
$\operatorname{diam} \tilde{j}_{0}^{n}(A)>n+1$. Pick $k_{n}$ sufficiently large to guarantee that if $\alpha_{n}: S^{k} \rightarrow X_{n} \times$ $Q$ represents $\left[\omega_{n}\right]$, then $\tilde{\alpha}_{n}: S^{k} \rightarrow \tilde{X}_{n} \times Q$ has diam $\tilde{\alpha}_{n}\left(S^{k}\right)>M_{n}$. This can be done since $\operatorname{diam} \tilde{\alpha}_{n}\left(S^{k}\right) \geqslant 2 k_{n}$. Thus, diam $\tilde{j}_{0}^{\tilde{n}} \tilde{\alpha}_{n}\left(S^{k}\right)>n+1$. Suppose there is a map $\alpha$ : $S^{k} \rightarrow Y$ representing the constructed shape morphism $\omega$. We can set $\alpha_{n}=j_{n} \alpha$ and conclude that

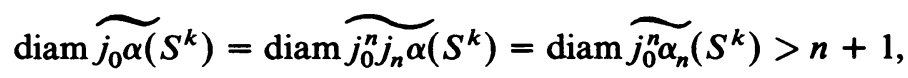

for each $n$. This contradicts the compactness of $S^{k}$ and completes the proof.

\section{Appendix.}

LEMMA. Let $A=\lim A_{n}$ with bonds $p_{n}: A_{n} \rightarrow A_{n-1}$ and with each $A_{n}$ a compact ANR. If $X$ is a compactum shape equivalent to $A$ then there is a sequence of embeddings $j_{n+1}: A_{n+1} \times Q \rightarrow A_{n} \times Q$ such that $X=\lim _{\leftarrow}\left(A_{n} \times Q, j_{n}\right)=$ $\cap_{n=1}^{\infty} j_{1}^{n}\left(A_{n} \times Q\right)$. Moreover, $j_{n}$ is homotopic to $p_{n} \times \mathrm{id}_{Q}$.

Proof. If $j: A \times Q \rightarrow Q$ is a Z-embedding, then $j(A \times Q)=\cap_{i-1}^{\infty} M_{i}$, where each $M_{i}$ is a $Q$-manifold neighborhood of $A \times Q$ homeomorphic to $A_{i} \times Q$ in such a way that the diagrams

$$
\begin{array}{ccc}
M_{i+1} & \rightarrow & M_{i} \\
\downarrow \simeq & & \downarrow \simeq \\
A_{i+1} \times Q & \stackrel{p_{i+1} \times \mathrm{id}_{Q}}{\rightarrow} & A_{i} \times Q
\end{array}
$$

commute up to homotopy. This is well known. See [1], for example, for a proof.

Since $A \times Q$ and $X$ are shape equivalent, the proof of Chapman's complement theorem [1] produces an isotopy $f_{t}: Q \rightarrow Q, 0 \leqslant t<1$, such that $f_{t}$ and $f_{t}^{-1}$ are supported on smaller and smaller neighborhoods of $A \times Q$ and $X$, respectively. If $\left\{t_{i}\right\}$ is a sequence of real numbers, $0 \leqslant t_{i}<1$, converging rapidly to $1, X=$ $\bigcap_{i=1}^{\infty} f_{t_{i}}\left(M_{i}\right)$ and $f_{t_{i}} \mid M_{i+1}$ is ambient isotopic to $f_{t_{i+1}} \mid M_{i+1}$ in $M_{i}$. This shows not only that neighborhoods of $A$ are homeomorphic (simple homotopy equivalent) to neighborhoods of $X$ but also that the homeomorphisms can be chosen coherently.

\section{REFERENCES}

1. T. A. Chapman, Lectures on Hilbert cube manifolds, CBMS Regional Conf. Ser. in Math., no. 28, Amer. Math. Soc., Providence, R.I., 1976.

2. S. Ferry, A stable converse to the Vietoris-Smale Theorem with applications to shape theory, Trans. Amer. Math. Soc. 261 (1980), 369-386.

Department of Mathematics, University of Florida, Gainesville, Florma 32611

Current address: Mathematical Reviews, 611 Church Street, Ann Arbor, Michigan 48109 